\title{
Incremental Value of Three-Dimensional Echocardiography over Two-Dimensional Echocardiography in the Assessment of Atrial Septal Defects
}

\author{
Charles German ${ }^{1}$, Navin C. Nanda ${ }^{2}$ \\ ${ }^{1}$ Department of Internal Medicine, University of Alabama at Birmingham, Birmingham, Alabama \\ USA, ${ }^{2}$ Division of Cardiovascular Disease, University of Alabama at Birmingham, Birmingham, \\ Alabama, USA
}

(Cardiovasc. j. 2015; 7(2): 67-71)

Congenital heart disease (CHD) affects approximately 6 to 13 newborns per 1000 live births, ${ }^{1}$ and is comprised of 5 major atrial septal defects (ASDs), including primum and secundum type defects, coronary sinus and sinus venosus defects, and patent foramen ovale (PFO). However, there is debate within the medical community as to whether a PFO should be included due to the fact that PFOs do not have absent septal tissue and typically result from separation of the primum septum from the secundum septum when the pressure in the right atrium (RA) exceeds the pressure in the left atrium (LA) forming a right to left shunt. Likewise, coronary sinus defects (unroofed coronary sinus) may not be considered true septal defects as they represent an abnormal communication between the superior portion of the coronary sinus and the bordering LA. Even sinus venosus defects may not be considered true septal defects because they result from straddling of the superior vena cava (SVC), or in rare cases of the inferior vena cava (IVC), in relation to the interatrial septum (IAS). Regardless, these defects can go undiagnosed at birth if asymptomatic, and may be found unexpectedly on routine imaging. And in instances of critical life threatening CHD, quick and efficient diagnosis via echocardiography is paramount in establishing the optimal treatment and ultimately improving mortality.

Secundum defects are the most common type of ASDs, and occur twice as often in females compared to males. $^{2}$ They classically involve the middle portion of the IAS but can occur in other areas of the IAS and result from either excessive absorption of the septum primum or arrested growth of the septum secundum. Two-dimensional echocardiography (2DE) is adequate in detecting these defects, but the images obtained can lack the detail necessary to precisely measure their size, shape, and location. This is most pronounced when assessing the multiple holes or Swiss cheese pattern, in which these limitations become more evident. Three-dimensional transthoracic echocardiography (TTE) performed via the apical, para-apical, right parasternal and subcostal views provide enhanced visualization of the defect in the majority of patients (Fig 1). Furthermore, 3D TTE has been shown to better approximate the dimensions and locations of ASDs as well as the adjoining anatomy and rim size as compared to 2D images. ${ }^{3,4}$ In one study, Morgan et al. compared parameters obtained from 2D transesophageal echocardiography (TEE) to 3D TTE. Although the differences in measuring the defect's circumference, area, and diameter was not statistically significant when comparing the two imaging studies, it was clinically significant, showing that 3D TTE was just as accurate in its ability to recognize appropriate candidates for percutaneous closure of ASDs, there by reducing the need for the more invasive TEE procedure and bypassing its major complications such as gastrointestinal bleeding, esophageal hematoma formation, and perforation. ${ }^{5}$

Address of Correspondence : Charles German, Department of Internal Medicine, University of Alabama at Birmingham, Birmingham, Alabama USA. 

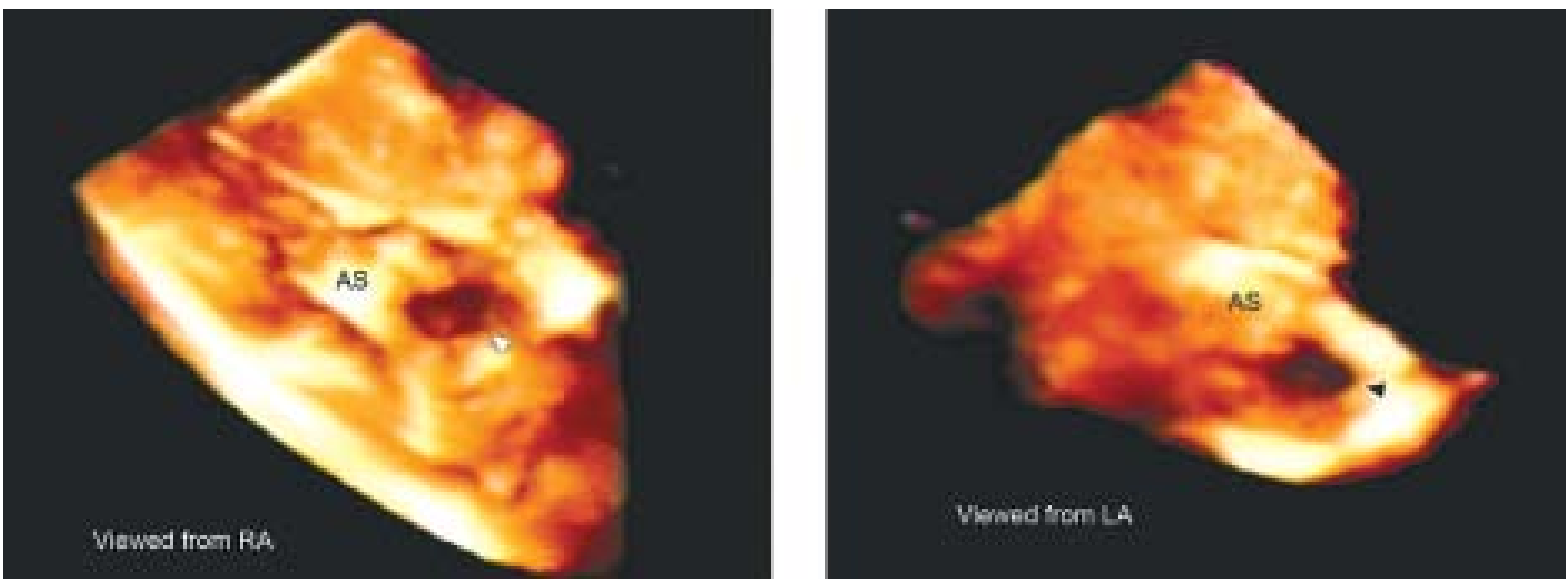

Fig.-1 a \& b: Three-dimensional transthoracic echocardiographic (3D TTE) assessment of atrial septal defect. Arrowhead points to a large secundum atrial septal defect visualized from both right (RA) and left atrial (LA) aspects. Note the large rim of tissue surrounding the defect. (AS: Atrial septum).(Reproduced with permission from Mehmood F, Vengala $S$, Nanda NC, et al. Usefulness of live three-dimensional transthoracic echocardiography in the characterization of atrial septal defects in adults. Echocardiography J. 2004;21:707-713).

Most ASDs, including the secundum type, have historically been repaired via median sternotomy, though advancements in technology have made percutaneous closure the current treatment of choice in the majority of patients. This decision is based on the specifications of the defect, including its size, shape, and location in space compared to the surrounding tissues. ${ }^{3,6}$ Percutaneous transthoracic repair can be done if the rim size is greater than $5 \mathrm{~mm}$. Thus, it is important to correctly obtain a complete image of the defect in order to ascertain its eligibility for the less invasive procedure. The ability of 3D TTE to approximate measurements precisely not only helps when planning the transcatheter approach to the procedure, ${ }^{3,7}$ but also provides important data required to select the appropriate size of the occluder device which is crucial. Selecting a poorly fitting occluder device can lead to major complications, including breakdown of the device, persistence of the shunt, device embolization, and even perforation of the heart. ${ }^{8}$ Additionally, 3D TTE has also been shown to help in detecting the above-mentioned complications, allowing for immediate recognition and reversal of the complication, leading to speedy repair and recovery. ${ }^{9}$ For example, color Doppler 3D TTE can unearth residual shunt after implantation of an
ASD occluder and can aid in determining the efficacy of other percutaneous closure devices used for ASDs as well as PFOs. ${ }^{10}$

Atrioventricular septal defects (AVSD) result from incomplete synthesis of the superior and inferior endocardial cushions during fetal development, resulting in irregular development of the atrioventricular septum and valves, ${ }^{12}$ leading to both atrial and ventricular septal defects. Primum type ASDs are considered to be within the realm of AVSDs, as their pathophysiology is related to faulty fusion of the endocardial cushions with the IAS. AVSDs can be classified as complete, intermediate, and partial. Complete AVSDs can be further grouped based on the superior bridging leaflet and its attachments to the crest of the ventricular septum and right ventricle via the Rastelli system, ${ }^{13}$ which provides important surgical and prognostic information. When measuring the specifications of AVSDs and adjacent cardiac structures, 3D TTE has proven to be far more accurate than $2 \mathrm{D}$ TTE. ${ }^{11}$ For example, 3D TTE can provide a detailed depiction of the characteristic five leaflets in complete defects (Fig 2), and can easily recognize the superior bridging leaflet and its attachment used to categorize ASVDs into the modified Rastelli types. ${ }^{11,13}$ 

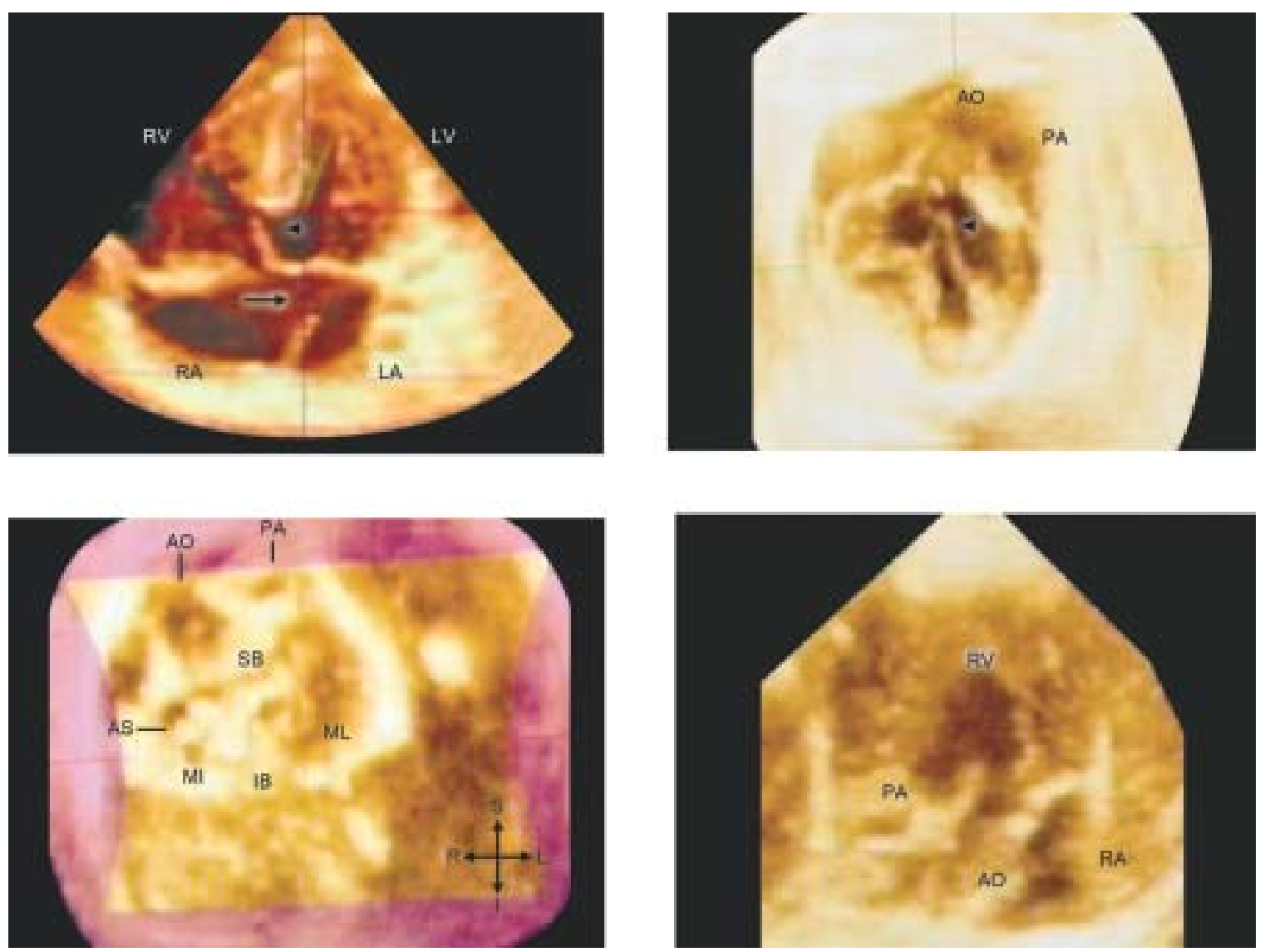

Fig.-2 a-d: Three-dimensional transthoracic echocardiography (3D TTE) in atrioventricular septal defects (AVSDs). (a) Complete AVSD. Arrowhead shows attachment of the common atrio-ventricular valve (CAV) to the crest of the ventricular septum (Rastelli type A). Arrow points to atrial component of the defect; (b) Complete AVSD. Arrowhead points to an anomalous papillary muscle projecting into the left ventricular outflow tract causing subaortic obstruction; (c and d) Intermediate AVSD; (c) En face view of CAV shows superior bridging (SB) leaflet crossing over into the $R V$; (d) Both the $A O$ and the PA are seen arising from the $R V$ consistent with double outlet right ventricle. (AS: Anterosuperior leaflet; IB: Inferior bridging leaflet; L: Liver; LA: Left atrium; LV: Left ventricle; MI: Mural inferior leaflet; ML: Mural lateral leaflet; PA: Pulmonary artery; PV: Pulmonary valve; RA: Right atrium; RV: Right ventricle; RAV: Right atrioventricular valve; RVO: Right ventricular outflow tract; SB: Superior bridging leaflet). (Reproduced with permission from Singh A, Romp RL, Nanda NC, et al. Usefulness of live/real time three-dimensional transthoracic echocardiography in the assessment of atrioventricular septal defects. Echocardiography 2006;23:598-606).

Sinus venosus ASDs (SVASD) are uncommon and form only $5 \%$ as opposed to secondum defects which comprise $75 \%$ of all ASDs. These defects are noted by echocardiography at the junction of the SVC, or rarely IVC, with the IAS. Unlike TEE, they are difficult to diagnose by TTE in an adult because the region of SVC entrance into the RA is not generally imaged during a routine $2 \mathrm{D}$ echo study. Therefore, non-standard approaches such as the right parasternal window are used to detect them. While both 2D and 3D echocardiography have been shown to be effective in detecting SVASDs, the latter has proven to be advantageous. 3D TEE can correctly visualize the defect in relation to the SVC and the anomalously draining right superior pulmonary vein while $2 \mathrm{D}$ imaging has been inferior in this regard (Fig 3) and can also calculate the area of SVASDs due to the added dimensions gained from the $3 \mathrm{D}$ rendered image. ${ }^{14}$ 

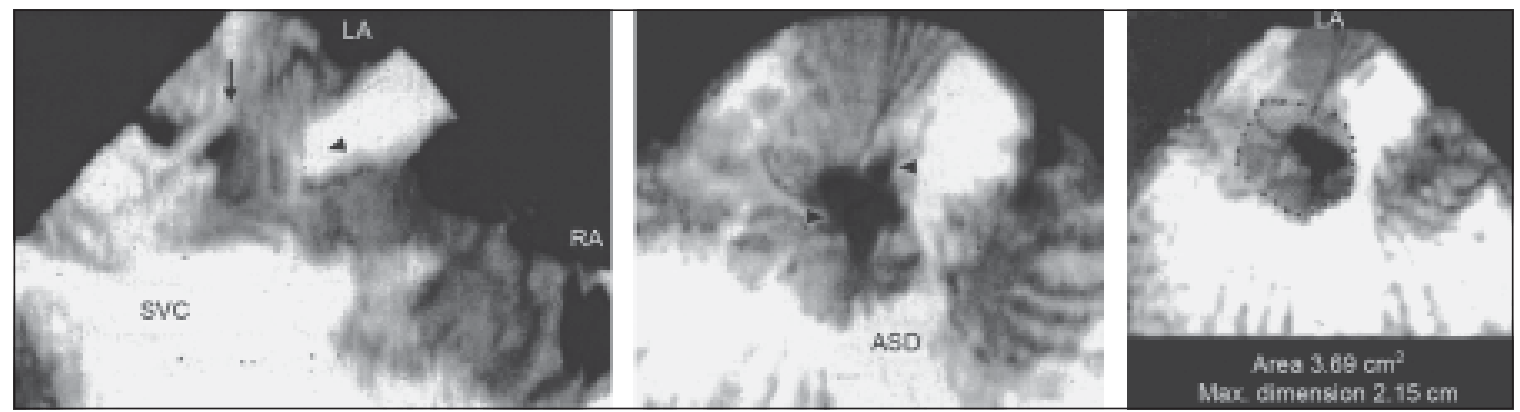

Fig.-3 a,b,c: Multiplane three-dimensional transesophageal echocardiographic reconstruction of sinus venosus atrial septal defect. (a) The arrowhead points to the large defect in the superior portion of the atrial septum. The arrow shows the right superior pulmonary vein entering the SVC-atrial junction at the site of the defect. ( $b$ and c) Orthogonal views demonstrating the size of the defect (ASD), which measured $3.69 \mathrm{~cm}^{2}$ in area. The maximal dimension of the defect was $2.15 \mathrm{~cm}$, which corresponded to the diameter of $2 \mathrm{~cm}$ measured at surgery. The top arrowhead in B points to the right superior pulmonary vein, and the bottom arrowhead points to the defect. (ASD: Atrial septal defect; SVC: Superior vena cava; LA: Left atrium; RA: Right atrium).(Reproduced with permission from Nanda NC, Ansingkar K, Espinal $M$, et al. Transesophageal three-dimensional echo assessment of sinus venosus atrial septal defect. Echocardiography. 1999;16:835-837).

Briefly, PFOs typically occur, as mentioned previously, through separation of the septum primum from the septum secondum due to elevated RA pressure, forming a shunt. A specific diagnosis can be made if a saline bubble study during the Valsalva maneuver, straining or abdominal pressure (more convenient during TEE) shows bulging of the IAS into the LA with micro bubbles crossing the PFO region from the RA into the LA. Transient bulging of the IAS into the LA suggests that the maneuver was successful in raising the RA pressure higher than the LA pressure. Thus, a PFO can be missed, but also cannot be excluded in the absence of IAS bulging. PFOs may be better diagnosed by 2D TEE as compared to 2D TTE because of its superior image quality. A PFO can also be missed during TTE or TEE unless there is a good contrast effect with complete filling of the RA. But 3D TEE may supplement 2D TEE by enabling a more detailed description of the complete PFO anatomy and surrounding tissues to a potentially higher degree of certainty, which can be helpful in differentiating intracardiac from extracardiac shunts. ${ }^{15}$

In conclusion, echocardiography has been crucial as a diagnostic tool for all forms of CHD, including ASDs. Once the diagnosis is clear, imaging further helps delineate the best treatment option. While $2 \mathrm{DE}$ is still recommended by current guidelines as the gold standard, it is evident that 3DE can provide valuable supplemental information. The major advantages of $3 \mathrm{DE}$ over $2 \mathrm{DE}$ are en face views of the defects with clear delineation of rim tissue as well as the surrounding structures and more accurate measurements of defect dimensions and areas. ${ }^{16}$ In addition, since the $3 \mathrm{D}$ data set containing the defect and the surrounding structures can be stored in a convenient medium such as a compact disc or an external drive, it can be re-cropped and sectioned in any desired manner. These findings can then be reviewed again for accuracy by either the same or different observers at any time, which is not possible with 2DE.As the technology develops further and becomes more widespread, 3DE use will potentially lead to better discovery of anomalies, reduction in repair complications rates, and ultimately enhanced patient care.

\section{References}

1. Ferencz C, Rubin JD, McCarter RJ, et al. Congenital heart disease: prevalence at live birth. The BaltimoreWashington Infant Study. Am J Epidemiol 1985; 121: 31-36.

2. Helgason H, Jonsdottir G. Spontaneous closure of atrial septal defects. Pediatr Cardiol 1999; 20:195-199.

3. Marx GR, Fulton DR, Pandian NG, et al. Delineation of site, relative size and dynamic geometry of atrial septal defects by real time three-dimensional echocardiography. J Am Coll Cardiol 1995;25(2):482-490. 
4. Sasaki T, Miyasaka Y, Suwa Y, et al. Real time threedimensional transesophageal echocardiographic images of platypnea-orthodeoxia due to patent foramen ovale. Echocardiography 2013;30(4):E116-117.

5. Magni G, Hijazi ZM, Pandian NG, et al. Two- and threedimensional transesophageal echocardiography in patient selection and assessment of atrial septal defect closure by the new DAS-Angel Wings device: initial clinical experience. Circulation 1997;96(6):1722-1728.

6. De Castro S, Caselli S, Papetti F, et al. Feasibility and clinical impact of live three-dimensional echocardiography in the management of congenital heart disease. Echocardiography 2006;23(7):553-561.

7. Dod HS, Reddy VK, Bhardwaj R, et al. Embolization of atrial septal occluder device into the pulmonary artery: a rare complication and usefulness of live/real time three-dimensional transthoracic echocardiography. Echocardiography 2009;26(6):739-741.

8. Berdat PA, Chatterjee T, Pfammatter JP, et al. Surgical management of complications after transcatheter closure of an atrial septal defect or patent foramen ovale. J Thorac Cardiovasc Surg 2000;120(6): 1034-1039.

9. Sinha A, Nanda NC, Misra V, et al. Live threedimensional transthoracic echocardiographic assessment of trans- catheter closure of atrial septal defect and patent foramen ovale. Echocardiography 2004;21(8):749-753.

10. Wei J, Hsiung MC, Tsai SK, et al. Atrial septal occluder device embolization to an iliac artery: a case highlighting the utility of three-dimensional transesophageal echocardiography during percutaneous closure. Echocardiography 2012;29(9):1128-1131.

11. Singh A, Romp RL, Nanda NC, et al. Usefulness of live/ real time three-dimensional transthoracic echocardiography in the assessment of atrioventricular septal defects. Echocardiography 2006;23(7):598-608.

12. Nanda NC, Ansingkar K, Espinal $M$, et al. Transesophageal three-dimensional echo assessment of sinus venosus atrial septal defect. Echocardiography 1999;16(8):835-837.

13. Singh P, Mehta A, Nanda NC. Live/real time threedimensional transthoracic echocardiographic findings in an adult with complete atrioventricular septal defect. Echocardiography 2010;27(1):87-90.

14. Ootaki Y, Yamaguchi M, Yoshimura N, et al. Unroofed coronary sinus syndrome: diagnosis, classification, and surgical treatment. J Thorac Cardiovasc Surg 2003;126(5): 1655-1656.

15. Shanks M, Manawadu D, VonderMuhll I, Khan K, Becher H, Choy J. Detection of Patent Foramen Ovale by 3D Echocardiography. J Am Coll Cardiol Img 2012;5(3):329-331.

16. Nanda NC, Abd-El Rahman SM, Khatri G, Agrawal G, El-Sayed AA, Hassanian HA et al. Incremental value of three-dimensional echocardiography over transesophageal multiplane two-dimensional echocardiography in qualitative and quantitative assessment of cardiac masses and defects. Echocardiography 1995;12:619-628. 